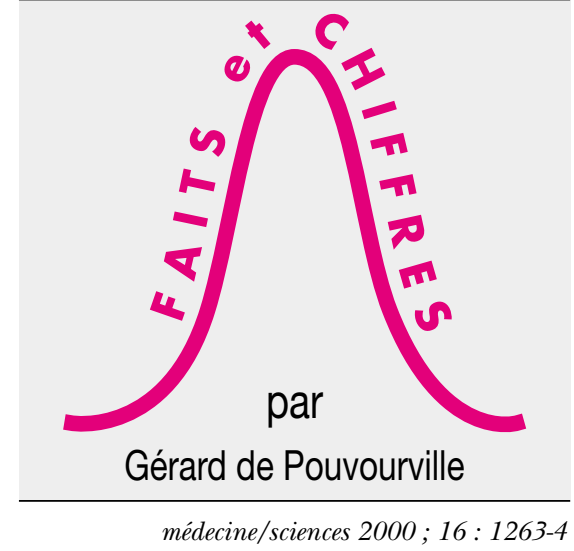

$\mathbf{L}$ es lecteurs de médecine/sciences sont maintenant familiers des concepts de base des Comptes nationaux de la santé. Voici les enseignements principaux de l'année 1999 (Tableau I).

On reste sur un rythme régulier de croissance des dépenses de soins de santé. Cependant, la croissance économique est de retour, et la part des deux grands agrégats dans la richesse nationale, mesurée par le PIB, est quasi-constante : 8,7 \% du PIB pour la consommation médicale totale, 9,5\% pour la dépense courante de santé. Les années passant, un autre résultat traduit une première tendance : depuis 1995, la part de la consommation médicale totale dans la consommation globale des ménages est stable, à 12,3\%. Cette part avait augmenté régulièrement, traduisant l'importance grandissante prise par les soins de santé.

\section{La structure de la consommation}

L'analyse de la répartition de ces dépenses entre les différents postes

\title{
Les comptes nationaux de la santé 1999 : des tendances se dessinent...
}

permet là aussi de déceler des tendances intéressantes. En effet, la part prise par l'hôpital dans la consommation de soins et de biens médicaux ne cesse de décroître depuis dix ans. Elle est passée de 48,4\% en 1990 à 46,8 \% l'an passé. Il en va de même des dépenses de soins ambulatoires, qui couvrent principalement les recours aux médecins, auxiliaires médicaux et dentistes, dont la part passe de 28,2\% en 1990 à 26,6\% en 1999. En faveur de quels postes la redistribution se fait-elle? D'abord en faveur du médicament remboursable, qui représente maintenant $20 \%$ du total, dont le montant augmente de $6 \%$ pour la deuxième année consécutive. En moyenne, chaque français dépense $2500 \mathrm{~F}$ par an de médicaments. Le rapport de la Commission des Comptes de la Santé donne par ailleurs un résultat fort instructif: les médicaments génériques représentent cette année $4 \%$ des volumes vendus, contre 3,2\% l'an passé. La part de ces produits, dont le prix moyen se situe environ $30 \%$ plus bas que les spécialités, reste donc modes-

Tableau I

DÉPENSES DE SOINS DE SANTÉ

\begin{tabular}{|l|c|c|}
\hline & $\begin{array}{c}\text { Dépense courante } \\
\text { de santé 1999 }\end{array}$ & $\begin{array}{c}\text { Consommation médicale } \\
\text { totale 1998 }\end{array}$ \\
\hline Total en MF & 871405 & 742600 \\
\hline Per capita en francs & 14523 & 12600 \\
\hline Évolution 1999/1998 & $3,5 \%$ & $3,6 \%$ \\
\hline Évolution 1998/1997 & $\mathbf{4} \%$ & $\mathbf{4 , 1} \%$ \\
\hline
\end{tabular}

te. Enfin, le dernier poste dont la part augmente est celui des autres biens médicaux (optique, prothèses et orthèses, véhicules pour handicapés, petits matériels et pansements) : les dépenses sont passées de 3,7\% du total en 1990 à 5,1 \% l'an passé.

\section{L'activité et les dépenses}

hospitalières

Les dépenses du secteur hospitalier public continuent de croître, mais à un rythme inférieur à celui de 1998 . Elles représentent environ $5500 \mathrm{~F}$ par habitant. En revanche, et ce fait est notable, les dépenses du secteur hospitalier privé ont décru en 1999. Lorsque l'on se rapporte aux données d'activité, on retrouve cette redistribution des parts des deux secteurs : le nombre d'entrées continue de croître dans le public, diminue dans le secteur privé. Selon les rédacteurs du rapport des Comptes de la santé, cela traduit un transfert de l'activité d'obstétrique du privé vers le public, mais aussi l'accroissement de l'activité des établissements de long séjour, qui appartiennent principalement au secteur public. La durée moyenne de séjour continue de baisser dans les deux secteurs. Nous disposons cette année de quelques indicateurs de volume par nature d'activité sur l'année 1998. Par exemple, on comptabilise en 1998 environ 11 millions de passages aux urgences, dont $90 \%$ dans le secteur public. Autrement dit, en moyenne, un français sur six se présente aux urgences hospitalières au cours de l'année. Il y a eu en tout 3,3 millions de séances de dialyses pour insuffisance rénale. Le secteur privé réalise les 


\begin{tabular}{|l|c|}
\hline \multicolumn{2}{|c|}{ Tableau II } \\
\hline DÉPENSES DE SOINS AMBULATOIRES \\
\hline Prestataires & Montant per capita (en francs) \\
\hline Médecins & 1563 \\
\hline Auxiliaires médicaux & 643 \\
\hline Dentistes médicaux & 682 \\
\hline Analyses & 285 \\
\hline Cures thermales & 125 \\
\hline
\end{tabular}

\begin{tabular}{|l|c|}
\hline \multicolumn{2}{|c|}{ Tableau III } \\
\hline HONORAIRES MOYENS PAR PRESTATAIRES (1998) \\
\hline Omnipraticiens & Montant per capita (en francs) \\
\hline Spécialistes & 646000 \\
\hline Dentistes & 1131000 \\
\hline Infirmiers & 995000 \\
\hline Kinésithérapeutes & 420000 \\
\hline
\end{tabular}

deux tiers de ces séances. Sur l'ensemble de ces séances, les autodialyses représentent environ $25 \%$, et les dialyses à domicile à peine $7 \%$. Leur part est en diminution au profit principalement des autodialyses. Le nombre total d'accouchement a été de 727200 en 1998, dont $16 \%$ environ avec césarienne.

\section{Les dépenses de soins ambulatoires}

Elles représentent environ $3300 \mathrm{~F}$ par habitant. Les honoraires de médecins en forment la plus grosse part, envi- prévention ou de santé publique (Tableau IV), pour donner un ordre de grandeur des montants investis dans ce type de prestations. Ces dépenses ont connu une augmentation annuelle de 4,9\% en 1999 par rapport à 1998 .

\section{L'évolution du financement des dépenses de soins de santé}

Les dépenses courantes de soins et de biens médicaux sont financées à hauteur de 76,7\% par la Sécurité Sociale et l'État. La part directe de financement par les ménages reste stable, à $11,1 \%$. Cependant, cette couverture par des financements collectifs couvre des différences importantes entre l'hôpital et les soins ambulatoires. Les soins hospitaliers sont couverts par des financements collectifs à hauteur de 91,3\%, les dépenses de soins ambulatoires à hauteur de $66,9 \%$. Sur les trois dernières années, on observe une certaine stabilisation de la part des dépenses de santé couverte par des financements collectifs.

ron $48 \%$. Ces $3300 \mathrm{~F}$ se décomposent de la façon suivante en fonction de la nature des prestations (Tableau II).

Le tableau suivant présente le montant moyen correspondant d'honoraires perçu par prestataires en 1998. Il s'agit d'un chiffre d'affaires. Pour obtenir un revenu avant impôt, il faut retrancher les charges professionnelles (Tableau III).

\section{Les dépenses de prévention}

On présente ici les montants des dépenses par nature d'activité de En conclusion, la hausse des dépenses de santé continue en 1999, mais à un rythme parallèle à celui de la richesse nationale. La part des dépenses de santé dans la consommation des ménages se stabilise, mais on observe une redistribution des dépenses entre les différentes prestations, principalement vers les médicaments et les produits médicaux divers, au détriment des dépenses de soins à l'hôpital et en ville.

\section{Gérard de Pouvourville}

Cregas, Inserm/Cnrs, Centre de recherche en économie et gestion appliquée à la santé, Inserm U. 537, Pavillon de la Force, Hôpital Bicêtre, 80, rue du Général-Leclerc, 94276 Le KremlinBicêtre, France. 